\title{
LUGAR E IMPORTANCIA DEL PENSAMIENTO DE G. H. MEAD EN LAS RECIENTES CIENCIAS COGNITIVAS
}

\author{
Leopoldo Márquez Velasco \\ Pontificia Universidad Católica de Chile
}

\begin{abstract}
Resumen: El filósofo pragmatista George Herbert Mead, reconocido como fundador del interaccionismo simbólico en sociología, ha comenzado a aparecer sistemáticamente en discusiones recientes dentro de las ciencias cognitivas. Este trabajo explora y reseña las principales intervenciones del autor en temas como la cognición corporizada (embodied cognition), las neuronas espejo y el lenguaje. El objetivo es hacer una puesta al día sobre las distintas líneas de investigación en la teoría de la cognición meadiana y proponer como característica valiosa fundamental del autor su carácter transdisciplinario y su constante interés en vincular los aspectos más teóricos de las ciencias de la mente con cuestiones prácticas sociales.
\end{abstract}

Descriptores: Mead · Ciencias Cognitivas · Cognición Corporizada · Neuronas Espejo · Pragmatismo $\cdot$ Lenguaje y Cognición.

Abstract: George Herbert Mead has started to appear systematically in recent discussions on cognitive sciences. This paper reviews and explores the main approaches of the author on embodied cognition, mirror neuron, and language. My goal is to update different lines of research in the Meadian theory of cognition. Additionally, I propose that his most important contributions to the field are the transdisciplinary character of his thought and his constant interest in linking the most theoretical aspects of cognitive sciences with practical social issues.

Keywords: Mead · Cognitive Science · Embodied Cognition · Mirror Neuron · Pragmatism · Language and Cognition.

Enviado: 23/05/2019. Aceptado: 17/09/2019

George Herbert Mead (1863-1931) fue un psicólogo y filósofo estadounidense del pragmatismo clásico asociado a la escuela sociológica de Chicago y considerado el fundador del interaccionismo simbólico en sociología (Blumer, 1969). Estudió con J. Royce, W. Wundt, y W. Dilthey y fue colega de J. Dewey y J. Watson, entre otros. Trabajó en la Universidad de Chicago desde finales del siglo XIX hasta su muerte en 1931. Aunque nunca escribió un libro, sus discípulos editaron cuatro volúmenes tras su muerte, recopilando notas y manuscritos del autor con notas de los estudiantes y taquigrafías de algunos de sus cursos en Chicago (Mead, 1932, 1934, 1936, 1938). 
Posteriormente han aparecido más volúmenes de este tipo: (Mead, 1982, 2011). E1 volumen más famoso e influyente es Mind, Self, and Society (Mead, 1934) basado en su célebre curso de psicología social. Pero la mejor fuente de su filosofía está en sus artículos publicados en algunas compilaciones: (Mead, 1964, 2001, 2011). La filosofía general de Mead puede caracterizarse como un naturalismo no reductivo de corte social, antidualista y antimentalista. El núcleo de su pensamiento consiste en una explicación genética de la emergencia de la subjetividad. Considera a la mente, la conciencia y el sí-mismo (self) como emergentes de procesos sociales de coordinación y control. La explicación de este surgimiento se hace en un marco científico no reductivo en el que se privilegia la biología y la psicología social. De esta forma, la filosofía de Mead puede verse como un intento de mantener el punto de vista objetivo de la ciencia en relación con la subjetividad propia del sí mismo, pero se trata de una objetividad amplia: la objetividad del lenguaje, la actividad, las interacciones y la cultura (Koczanowicz, 2011, p. 66).

Desde finales del siglo pasado, ha habido en filosofía un revival del pragmatismo con cierto interés por los autores clásicos como C. S. Peirce, W. James, J. Dewey y G. H. Mead (Bernstein, 2010). Dicho interés también ha calado más recientemente en las ciencias cognitivas desde diversas perspectivas. A continuación, exploro y reseño brevemente las principales intervenciones de Mead en este terreno: su reciente aparición en la discusión sobre cognición corporizada, la transdisciplinariedad y el carácter social de su obra, la relevancia del fenómeno de las neuronas espejo, los límites y aplicaciones de su explicación lingüística, entre otras cuestiones. Todo lo cual refleja la relevancia de su pensamiento, especialmente en la reciente ampliación de la visión científico-cognitiva.

\section{MEAD COMO UN TEÓRICO DE LA CORPORIZACIÓN (EMBODIMENT)}

Hubiese sido muy distinto elaborar este trabajo en la década de los 70, cuando la ciencia cognitiva se basaba principalmente en el modelo computacional de la mente: un modelo mentalista, individualista y reductivo del pensamiento y la acción (Fodor, 1975, 1980). La concepción pragmatista, externista y social del pensamiento, el conocimiento y la acción que caracterizan a Mead habría calado como una crítica al dualismo, el mentalismo y el individualismo dominante. Las cosas han estado cambiando. Se habla ahora de una ciencia cognitiva de 'segunda generación' (Lakoff \& Johnson, 1999) o incluso de un 'giro pragmático' (Engel, Friston, \& Kragic, 2015) que comenzó en la década de los 90 con los trabajos de Varela, Thompson, Rosch (Varela, Thompson, \& Rosch, 1991), Lakoff, Johnson (Lakoff \& Johnson, 1999) y Clark (Clark, 1997), entre otros. Shaun Gallagher ha caracterizado al nuevo enfoque como 'las cuatro E' (Rowlands, 2010, p. 3), debido 
a que la cognición se entiende como embodied, embedded, enacted y extended (corporizada, incrustada, enactiva y extendida). Si usamos estos términos como criterios, se reconocerá que Mead cumple con cada uno de ellos. S. Gallagher incluso reconoce que Mead, en tanto pragmatista, llega a tener una "perspectiva más integrada" de estos componentes (Gallagher, 2014).

La corporización de Mead se evidencia en distintos aspectos de su obra, pero es clara en su teoría antimentalista de la percepción que vincula el sentido de la visión con los posibles contactos o manipulaciones del objeto:

Overt food, protective, reproductive, fighting processes, all are made up of such movements toward or away from possible contacts, and the success of the conduct depends upon the accuracy with which the distance stimulation leads up to appropriate contacts... our perception of physical objects always refers color, sound, odor, to a possibly handled substrate. (Mead, 1907, p. 63) (Énfasis agregado)

Resume su idea diciendo: "We look because we handle, and we are able to handle because we look" (Mead, 1907, p. 64). También dice: "The meaning of a chair is sitting down in it, the meaning of the hammer is to drive a nail-and these responses can be innervated even though not carried out." (Mead, 1934, p. 104). Según ilustra Roman Madzia, uno de los comentaristas más interesantes de Mead en la discusión contemporánea sobre las ciencias cognitivas: "Mead's concept of attitudes as neurophysiological items encoding perceptual objects as 'invitations to action' (...) can also be understood in terms of James J. Gibson's term 'affordances' or opportunities of behavior" (Madzia, 2016, p. 304).

La cognición extendida sostiene que los sistemas cognitivos se extienden más allá de los límites del organismo individual. Dice Mead: "the field or locus of any given individual mind must extend as far as the social activity or apparatus of social relations which constitutes it extends; and hence that field cannot be bounded by the skin of the individual organism to which it belongs" (Mead, 1934, p. 223). En efecto, Mead niega que el mundo experimentado esté ‘en la cabeza' o en el cerebro y afirma, en su lugar, que el papel del 'conductista' (hoy diríamos 'enactivista') es relacionar el mundo de la experiencia con el acto total del organismo:

Well, instead of assuming that the experienced world as such is inside of a head, located at that point at which certain nervous disturbances are going on, what the behaviorist does is to relate the world of experience to the whole act of the organism. (Mead, 1934, p. 111).

El enactvismo (que podría considerarse como un desarrollo específico de la concepción extendida de la cognición) hace énfasis en el acoplamiento (coupling) entre el organismo y su entorno, según el cual los agentes "enactúan” (enact) o hacen emerger 
(bring forth) un mundo mediante sus acciones como cuerpos históricos vivientes (Varela, Thompson, \& Rosch, 1991, p. 149). En uno de sus primeros artículos, Mead caracteriza al organismo como: "a system built up by action upon its environment" (Mead, 1894c, p. 175) y, como los enactivistas, sitúa la idea en el contexto de la teoría evolucionista. La enacción en Mead se deja ver aún mejor cuando considera que: "the study of consciousness from the standpoint of the organism inevitably led men to look at consciousness itself from the point of view of action" (Mead, 1934, p. 22). También es crucial el tipo de relación enactiva que Mead plantea con el entorno:

The distribution of meaning to the organism and the environment has its expression in the organism as well as in the thing, and that expression is not a matter of psychical or mental conditions. There is an expression of the reaction of the organized response of the organism to the environment, and that reaction is not simply a determination of the organism by the environment, since the organism determines the environment as fully as the environment determines the organs. (Mead, 1934, p. 129) (énfasis agregados)

Esta última idea de que el organismo determina el entorno tanto como el entorno determina al organismo puede leerse también en A. Damasio: "the environment is, in part, a product of the organism's activity itself' (Damasio, 1994, p. xvii).

El carácter incrustado de la cognición se nota de forma dramática con la concepción de Mead del desarrollo intrauterino, pues no ve ninguna necesidad de asumir en el embrión un proceso de desarrollo distinto del de un organismo cualquiera que responde y se adapta en relaciones de intercambio con su entorno: "This environment is the blood of the mother form, from which the embryonic cells get all their nourishment and stimulus for development" (Mead, 2001, p. 75). Cuando el individuo nace, se aplica el mismo principio, siendo el entorno socio-bio-cultural el medio en el que está incrustado. (Mead saca mucho rendimiento práctico a esta idea en temas educativos y sociales (Mead, 1898) (Mead, 1899a).)

La dimensión afectiva, otra propiedad relacionada con la corporización, es también destacable en Mead cuando dice: "Thought and volition develop and interpret the situation that is first of all emotional" (Mead, 2001, p. 6), y dice que los objetos sociales se 'construyen' a partir de la 'conciencia emocional', de la misma manera en que los objetos físicos se construyen de sensibilidad. Hay que recordar que Mead es pionero en el trabajo de las emociones desde el punto de vista de la psicología funcionalista (Mead, 1895b).

S. Gallagher denunciaba en 2014 que, con excepción de James, los pragmatistas eran raramente citados:

There is no mention of Peirce, Dewey, or Mead, for example, in Noë (2004), Thompson (2007) or Hutto and Myin (2013). Varela et al. make one general and 
undeveloped reference to pragmatism (Varela et al. 1991, $30-31$ ), but without direct relevance to the enactive aspects of this work. (Gallagher S. , 2014, p. 111)

Ello se debe, en parte, a que la nueva generación reconoció inicialmente como antecedente filosófico a la fenomenología, especialmente la de Merleau-Ponty (Varela, Thompson, \& Rosch, 1991). Sin embargo, los pragmatistas han comenzado a ser más considerados recientemente (Madzia \& Jung, 2016). El problema es que Mead ha sido el menos atendido: "the most neglected but at the same time arguably one of the most original philosophers of embodiment" (Madzia \& Jung, 2016, p. 11). De hecho, y debido muy probablemente a la influencia de Rorty (Rorty, 1979), algunos se han abocado a buscar en John Dewey inspiración y antecedentes, pero esto, aunque es de celebrar, sigue siendo injusto con Mead. El punto que favorece a Mead es que muchos de los temas que se vinculan con Dewey en la discusión contemporánea dentro de las ciencias cognitivas no son centrales en dicho autor:

If we keep in mind that Dewey explicitly stated that he took over his most important concepts in social theory from Mead (see Jane Dewey 1931, 313; Dewey 1939, 26), I think we have to consider Mead's absence from large parts of the literature on pragmatism and the cognitive sciences a major shortcoming (Nungesser, 2016, p. 254).

El trabajo sobre el papel de la corporización en Mead está apenas comenzando. Surge de inmediato cuestiones como qué tan corporizada es la teoría de Mead o de qué tipo de corporización se trata. A este respecto vale mencionar el trabajo de Ryan McVeigh (McVeigh, 2015) y de Kelvin Booth (Booth K., 2016a). Este último reconoce en Mead un teórico de la corporización e intenta desarrollar a partir de él la teoría de la mente corporizada de Mark Johnson (Johnson, 2007), según la cual: "meaning and all our higher functioning as growing out of and shaped by our abilities to perceive things, manipulate objects, move our bodies in space, and evaluate our situation" (Johnson, 2007, p. 1). Como hemos visto, esto es por lo menos coherente con algunas afirmaciones de Mead. Ryan McVeigh ha relacionado el pensamiento de Mead con la investigación en las teoría de prototipos, especialmente en lo que respecta a la idea de basic-level categories de Lakoff y Johnson (Lakoff \& Johnson, 1999) y ha concluido que "Research on basic-level categories directly supports the understanding of mind and self articulated by G.H. Mead in a number of interesting ways" (McVeigh, 2015, p. 49) y que "Placing these two historically separate bodies of work together [Mead and cognitive sciences] thus reveals the relevance that both fields have for each other" (McVeigh, 2015, p. 47). Sin embargo, McVeigh cree que Mead a "minimizado" la importancia de la corporización y que la investigación contemporánea como la de Lakoff y Johnson suplen con creces estos defectos (McVeigh, 2015, p. 49). 
Una posición parecida encontramos en Jessica Lindblom. Ella también cree que "today's embodied and situated approaches of cognitive science complement Mead's theory of the socially interactive mind" (Lindblom, 2012, p. 63), pero, al igual que McVeigh, sostiene que "current theories of embodiment need to move beyond their individualistic perspective and also consider interactions 'between agents and their social environment"' (Lindblom, 2012, p. 52). Pero la autora, aunque reconoce el aporte al nivel social que puede hacer Mead a la cognición corporizada, considera la postura del pragmatista como 'descorporizada' (disembodied), pues:

Although Mead considered humans as embodied biological persons in a social context, he failed to explicitly address the bodily aspects of social interactions (Farnell 1999). In summary, the emphasis on interactions between agents and their social surroundings offers little on the embodied nature of human social interaction and cognition, and is currently peculiar "disembodied" (Lindblom, 2012, p. 56).

Hasta ahora habíamos visto evidencia textual de que Mead compartía ciertos postulados de la cognición cognitiva corporizada, pero hemos hallado también cierta discrepancia de opiniones con respecto a esta evidencia. Por un lado, están los que, como Madzia y Jung, consideran a Mead posiblemente el filósofo más original de la corporización, a McVeigh, quien cree que Mead no hizo el énfasis suficiente en la corporización y a Linblom quien llega a decir que es "particularmente descorporizado". Con respecto a esta última postura, más negativa, hay que decir dos cosas: Primero, Lindblom cita en su bibliografía solo a Mind, Self, and Society, que es un texto póstumamente editado por Charles Morris y que se basa en el curso de psicología social que impartía Mead, con lo cual no es de extrañar que haga más énfasis en lo social que en lo corpóreo. Muchas de las citas que yo he traído como muestra del carácter corporizado de la filosofía de Mead son de artículos escritos por él mismo que no son por lo general tomados en cuenta. Segundo y más importante como vimos en la cita de Lindblom, ella se basa en un artículo llamado "Moving Bodies, Acting Selves" de B. Farnell (Farnell, 1999), ipero en este trabajo el autor se refiere a Margaret Mead (1901-1978), la antropóloga, no a George Mead!

La tensión presentada en el último párrafo obedece, por lo menos en parte (no cabe duda), a la oposición que naturalmente ofrecen entre sí las nociones de "cognición social" y "cognición corporizada". Puede argumentarse que Mead dedicó toda su vida a armonizar ambas cuestiones. La idea de que la cognición sea corporizada se traduce en su insistencia en que la orientación subjetiva del individuo debe jugar un papel en la constitución social del conocimiento. Su estrategia, como repasaremos en los próximos apartados, involucra una visión diacrónica de la experiencia que toma en cuenta el entramado social que permite el surgimiento y desarrollo de nuestras capacidades cognitivas. El fallo de muchos intérpretes en 
comprender este intento, tan intensamente presente en la obra de Mead, de superar de las dicotomías interno-externo o subjetivo-intersubjetivo, etc., es lo que sigue generando los malentendidos propios de las distintas lecturas reduccionistas. Es por ello que, más allá de las divergencias, en el fondo, los autores considerados están de acuerdo en que a la cognición corporizada no le vendría mal un poco de la corporización social de Mead, que es como se podría caracterizar su postura. Queda, como ya he dicho, un trabajo que hacer para evaluar el grado de corporización de Mead y sus posibles aportes en este campo más allá de su énfasis en lo social, que tocaremos a continuación.

\section{EL ASPECTO SOCIAL Y EL CARÁCTER TRANSDICIPLINARIO}

Aunque bien puede Mead considerarse un filósofo de la corporización, ese no es el motivo principal de toda su obra. Lo que resulta casi omnipresente y absolutamente determinante en el pensamiento de Mead es lo social. Llega a decir que "The primitive instincts of the human animal are practically all social (...) primitive consciousness even of the physical world is social, and only becomes a physical consciousness with the growing powers of reflection" (Mead, 2001, p. 3). No se trata simplemente de que el ser humano necesite de la sociedad para surgir, sino que es un asunto intrínseco a la naturaleza de la acción y el pensamiento humano. Es precisamente lo social lo que no parece haber sido lo suficientemente destacado en la cognición corporizada, o por lo menos esa es la impresión que dejan las consideraciones hechas por algunos intervencionistas meadianos. Por ejemplo, en su comparación entre Mead con las categorías de nivel básico de Lakoff y Johnson, McVeigh acusa a los últimos de tener muchos supuestos individualistas y ve en Mead la forma de subsanar la deficiencia. Además, cree que la teoría de Lakoff y Johnson no hace el énfasis suficiente en la constitución social del significado: "embodiment simply cannot account for the richness and diversity of conceptual categories on its own (...) basic-level concepts are meaningful because of their functional differentiation in society;" (McVeigh, 2015, p. 50).

La versión más reciente de Johnson (Johnson, 2007) también es cuestionada en estos términos por Booth, quien reclama el desarrollo de la dimensión social en la teoría de la mente y del significado, y comenta: "The body that creates meaning is not only an emotional, kinesthetic, and aesthetically experiencing body; the body that creates meaning is a social body" (Booth, 2016a, p. 2). Algo parecido también ha sido señalado por $M$. Weichold, quien cree que con Mead se puede extender el enactivismo al dominio de lo social de un modo novedoso (Weichold, 2017, p. 147) y por F. Nungesser, quien enfatiza: "the stronger claim that human agency is in itself an intersubjective process, which must be explained on the basis of the 
individual's constitutive embeddedness in human sociality" (Nungesser, 2016, p. 252), y reconoce el aporte de Mead a este respecto.

Sin embargo, dentro de las ciencias cognitivas también se ha estado dando un giro hacia lo social (Brothers, 1997) (Franks, 2010). Brothers dice: "the network of meanings we call culture arises from the joint activities of human brains" (Brothers, 1997, p. xii). A todas estas, mientras que Mead cayó en el olvido para filósofos y psicólogos, ha sido siempre figura destacada entre sociólogos. Esto implica un acercamiento de disciplinas que se han visto separadas, como la sociología y las ciencias del cerebro. McVeigh tiene de nuevo palabras muy certeras al respecto:

The intent, then, is to move away from the sense of "mutual antagonism"... that properly characterizes the current relationship between the social and natural sciences (epitomized most clearly by the sociology of science tradition) toward an anti-dualist, anti-reductive position that incorporates and builds from the strengths of each discipline... Rather than see the disciplines as independent and antagonistic, the time has come for integration and association - to create both a cognitively informed sociology and a sociologically informed cognitive science. (...) the work of G.H. Mead is an ideal place to start (Mcveigh, 2016, pp. 61-2).

En este sentido, Mead ha sido inspiración para la neurosociología de Franks, quien dice, por ejemplo, que "Mead and his brief comments on what we now call the brain do imply some bridges between neuroscience and traditional symbolic interaction which have not always been recognized" (Franks, 2010, p. 2). La vinculación entre el cerebro (al que Mead se refiere constantemente como "sistema nervioso central") y los productos sociales es muy estrecha y atendida por Mead antes de que comenzara a ser común debido, especialmente, según veremos, al fenómeno de las neuronas espejo. Mead aclara que:

While minds and selves are essentially social products, products or phenomena of the social side of human experience, the physiological mechanism underlying experience is far from irrelevant — indeed is indispensable - to their genesis and existence (Mead, 1934, p. 1).

En efecto, Mead tiene una postura fuerte sobre la idea de cerrar la brecha entre fisiología y sociología, llegando a afirmar en uno de sus primeros escritos no publicados llamado "Social Psychology as Counterpart to Physiological Psychology" que: "From a logical point of view a social psychology is strictly parallel to a physiological psychology." (Mead, 2001, p. 17). En este sentido, Mead también ha sido reconocido, junto con Dewey, en una reciente integración entre neurociencia y pragmatismo llamada neuropragmatismo (Solymosi \& Shook, 2013).

Queda pues establecido uno de los puntos más llamativos de Mead: su carácter transdiciplinario. A este respecto comenta Nungesser: 
One of the key strengths of Mead's work is its deeply transdisciplinary character. Because he largely abstained from boundary-work, Mead was able to integrate arguments from diverse fields such as philosophy; social developmental, and animal psychology; physiology; evolutionary theory; and physics. (Nungesser, 2016, pp. 252-3).

En una época como la nuestra, que ha sufrido las consecuencias de la hiperespecialización, su obra es capaz de integrar argumentos y conceptos de distintos campos y tendencias.

\section{DeSARROLLO, NEURONAS ESPEJO Y LENGUAJE}

La característica más conocida del pensamiento de Mead es su teoría del desarrollo de lo mental, con la que intenta naturalizar la idea de intencionalidad, mostrando cómo surge desde una intencionalidad pre-reflexiva de corte biológico en su vinculación con prácticas de control y coordinación sociolinguisticas. Madzia y Jung explican:

Mead (...) provides the right tools to solve this pressing problem of accounting for continuity between human (reflective) consciousness and consciousness of nonlinguistic creatures. The key to this solution is Mead's conviction that all bodily coping, mediated by attitudes (neurologically realized bodily dispositions encoding perceived objects in terms of possible reactions toward them), is inherently goaldirected (quasi-intentional) but, in most cases, does not involve mental content. Hence, elaborating on Mead's concept of attitudes, the notion of intentionality can be divided into "bodily-interactive" and "social-symbolic" intentionality. The former shows that intentionality is not primarily a property of the mind but of the active body and may serve as a conceptual ground for social (second-order) intentionality (Madzia \& Jung, 2016, p. 4).

El gran problema al que se enfrentan los teóricos de la psicología evolucionista es lo que se ha dado en llamar "el problema evolutivo del tiempo en la hominización" (Tomasello, 1999, p. 2 y ss.), esto es, cómo en un período tan corto de tiempo los humanos desarrollaron una dinámica conductual tan distinta a la de sus antepasados, apareciendo una nueva "escala del tiempo": la historia cultural (Nungesser, 2016, p. 255). Este problema crea la presión de explicar el surgimiento de la mente en base a un fenómeno que puede generar un cambio radical y acelerado en el pensamiento humano. A pesar de no contar con todos los avances científicos de nuestra época, Mead fue consciente de este problema. En Mind, Self, and Society dice: "the rate of development or evolution of human society, since the emergence of minds and selves out of the human social processes of experience and behavior, has been tremendously accelerated as a result of that emergence" (Mead, 1934, 
p. 227 en nota). La cuestión, entonces, es explicar la aparición de la mente y el sí-mismo de modo evolutivo. La teoría de Mead ha sido inspiración para uno de los más importantes teóricos en este campo: Michael Tomasello (Tomasello, 1999). Además, recientemente, diversos comentaristas han hallado confirmación o complementación de diversos puntos de su teoría con otras propuestas en la ciencia cognitiva como la de Merlin Donald (Donald, 1991); Timothy Gallagher sostiene que: "Mead's description is strikingly close to Merlin Donald's mimetic theory of speech origins" (Gallagher, 2016, p. 326). La comparación entre Mead y las teorías de Tomasello y Donald están siendo bien atendidas y se han comenzó a mostrar diversas formas de intercambio y complementación. Algunos consideran que la teoría de Mead, como cualquier teoría de la piscología evolucionista no tiene ningún valor explicativo, aduciendo algunos quizá un innatismo del lenguaje (Bergesen, 2004). Pero aquellos interesados en una explicación del desarrollo no les queda más que destacar en Mead un precursor valioso en este campo y, aunque la teoría tiene algunas deficiencias o baches explicativo que teorías más recientes pueden subsanar, su originalidad y creatividad en las explicaciones sigue siendo reconocida y aplicada (Booth, 2016a) (Nungesser, 2016).

En el fenómeno de las neuronas espejo, neurociencia y sociología parecen encontrarse de manera más evidente, pues, se argumenta, se trata de la base neurológica para los fenómenos sociales de la empatía y la imitación (Iacoboni, et al., 2005). Las neuronas del individuo 'reflejan' el comportamiento del otro, como si el observador estuviera realizando la acción. Se podría afirmar que el descubrimiento es en parte responsable de los esfuerzos actuales de pensar la cognición en términos sociales. De hecho, Franks lo considera una especie de confirmación empírica de la teoría del desarrollo de Mead (Franks, 2010, p. 204). El tema, sin embargo, es complejo, tanto en sí mismo como en relación con Mead. Por un lado, es cierto que una de las tesis más importantes de Mead en relación con lo social es que la percepción del otro es inmediata. Dicha tesis fue enunciada por primera vez en 1895 (Mead, 1895, p. 401) y es central en el desarrollo de su filosofía hasta su muerte (Cf. Mead, 1934, p. 138). Esto lo distancia de cierto escollo típico de la filosofía de la mente: el problema de las otras mentes, pues el punto de partida de Mead hace desparecer el este problema y se enfoca en la mente individual como un producto social (Mcveigh, 2016, p. 222). Ahora bien, el desarrollo de la mente en Mead presupone la reflexión y la conciencia, que sólo los humanos poseemos, así que no basta con la noción de imitación, que ha jugado un papel importante en las teorías evolucionistas de la mente. De hecho, Mead fue bastante crítico de dicha noción (Mead, 2001, pp. 67-72). Booth cree que esto no constituye un problema, sino que más bien apoya la idea de que "mimetic abilities precede and are responsible for the ability to take the attitude of the other" (Booth, 2016, p. 232) ('tomar la 
actitud del otro' es el concepto central de Mead en este punto). En efecto, cree que Mead puede explicar la conducta animal en general, sin necesidad de apelar a una 'teoría de la mente' (Booth, 2016, p. 235). Sin embargo, las neuronas espejo no son exclusivamente humanas, así que no pueden funcionar como biomarcardor de la competencia social humana. Madzia dice que hay buenas razones para creer que las neuronas espejo no representan condiciones suficientes para la imitación en un sentido robusto (Madzia, 2013, p. 206). Nungesser comenta correctamente, según creo, que "research on mirror neurons focuses on the neurophysiological basis of social interaction, communication, and empathy. It does not, however, look into the importance of sociocultural processes for the development of human cognition" (Nungesser, 2016, p. 254).

Para que pueda surgir la mente humana se requiere más que una capacidad imitativa, se requiere lenguaje. En este sentido, David McNeill tiene una teoría que se sirve de Mead y las neuronas espejo para explicar el surgimiento del lenguaje (McNeill, 2005). Un punto importante en la teoría de Mead es que "Gestures become significant symbols when they implicitly arouse in an individual making them the same responses which they explicitly arouse, or are supposed to arouse, in other individuals, the individuals to whom they are addressed;" (Mead, 1934, p. 47). A esto le bautiza McNeill como Mead's Loop (el bucle de Mead) (McNeill, 2005, p. 250), una capacidad no presente en el resto de los primates que, según él, viene a completar el circuito de las neuronas espejo. Este desarrollo teórico es una de las más claras aplicaciones de la teoría de Mead a la ciencia cognitiva contemporánea y un claro ejemplo de cómo puede ser relevante el tema de las neuronas espejo para el desarrollo de su pensamiento.

Sin embargo, no todos son optimistas sobre las potencialidades de Mead en relación con el lenguaje. En este sentido, se ha llamado la atención (especialmente los chomskianos) sobre lo que se ha dado en llamar 'el problema de la sintaxis en Mead' y que ha generado cierta polémica. Se dice que su teoría del desarrollo del lenguaje no puede explicar el surgimiento de la sintaxis, lo cual ha sido reconocido por diversos comentaristas (Joas, 1985, p. 117). El asunto es si puede ser complementado contemporáneamente, o si esta carencia es algo más profundo y fatal según ha dicho Bergesen (Puddephatt, 2011, p. 83). Puddephatt traza un paralelo entre Mead y Piaget y dice que se aplica a la discusión sobre el lenguaje más o menos lo mismo en relación con ambos (Piaget, 1980). Los supuestos de Piaget, como los de Mead son meramente con respecto a la función, y consideraría especulativas las afirmaciones de Chomsky sobre las estructuras innatas (Puddephatt, 2011, p. 94). Aunque los chomskianos critican la falta de estructura, los funcionalistas como Piaget y Mead les criticarían a su vez la falta de atención al significado (Piaget, 1980), alineándose con autores más contemporáneos como John Searle, quien ve al 
lenguaje en términos de actos de habla, contextualmente determinados en relación con intenciones comunicativas (Searle, 1969). En definitiva, la situación en relación con el lenguaje es esta: algunos creen que Mead no tiene oportunidad de dar una explicación del lenguaje debido a cuestiones de fondo, lo que haría todo su proyecto inviable (Bergesen, 2004), otros creen que sí tiene una carencia importante, pero que puede ser subsanada con desarrollos contemporáneos (Nungesser, 2016), y otros creen que sí tiene una explicación correcta sobre el habla (Gallagher, 2016) que ha servido de inspiración para teorías contemporáneas (Mcveigh, 2016).

\section{Conclusión}

Hemos visto el lugar e importancia que distintos investigadores han hallado en la obra de Mead para distintos ámbitos de la reflexión cognitiva contemporánea. Su crítica al dualismo, el mentalismo y el individualismo siguen vigentes y su estrategia al abordar estos temas desde una perspectiva social es muy sugerente y ha sido inspiradora. Su obra está alineada con las tendencias más recientes de la cognición 4E y debe reconocerse como un antecedente valioso para dicha corriente, que está tomando en cuenta el aporte del pragmatismo como una opción alternativa a las recientes intervenciones fenomenológicas. El modo como hallamos en Mead todos los componentes propios de la cognición corporizada es original y se esperan futuros trabajos que revelen el aporte específico de su filosofía en este respecto.

El carácter transdiciplinario del pensamiento de Mead busca establecer una continuidad entre la investigación psicológica y la sociológica. En este sentido, hemos visto como distintos comentaristas destacan la visión social del desarrollo cognitivo que tiene Mead y que permitiría cerrar el abismo que a menudo se abre entre la experiencia propia de la perspectiva en primera persona y la dimensión impersonal e interpersonal de nuestro conocimiento. Esta es una línea de investigación aún no suficientemente explorada, pero estoy convencido de que Mead tiene recursos suficientes para armonizar la incorporación de una consciencia fenoménica subjetiva con una explicación de la constitución social del pensamiento, pues esto, puede argumentarse, fue el motivo principal de toda su filosofía y, según hemos visto, está presente desde sus primeros trabajos. A este respecto, hemos visto cómo la obra del pragmatista ha sido inspiración para teóricos del desarrollo como Tomasello y también cómo se incorpora su reflexión a la discusión contemporánea sobre las neuronas espejos y el lenguaje, donde hemos destacado la interesante aplicación que hace McNeill al respecto. Como ya se mencionó, aún queda trabajo por hacer en este tema.

La parte menos estudiada por la ciencia cognitiva y de especial importancia para la filosofía en general y la filosofía de la mente es la noción de social self de 
Mead. El trabajo de Mead sobre el sí-mismo y su lugar en la reflexión cognitiva y social no ha sido del todo recibida en las ciencias cognitivas ni en la filosofía de la mente, sin haber podido lograr lo que dijo J. Habermas que Mead lograba: "romper con el paradigma de la filosofía de la conciencia" (Habermas, 1987, p. 10). Lo más importante, según creo, de este concepto en Mead es la forma como permitiría vincular temas duros sobre la cognición como la neurociencia con asuntos de interés práctico general como la educación, la ética y la política, que lograrían expandir de manera unificadora el territorio de la explicación científica y humanística, muy parecido a como lo ha reclamado Franks u otros como Damasio:

Culture and civilization could not have arisen from single individuals and thus cannot be reduced to biological mechanisms, and even less, can they be reduced to a subset of genetic specifications. Their comprehension demands not just general biology and neurobiology but the methodologies of the social sciences as well. (Damasio, 1994, p. 124) [Énfasis agregado]

Esto es precisamente lo que trabajo Mead toda su vida y es en esta visión amplia y responsable que reside su más profundo valor. Es de esperar que futuras investigaciones desarrollen este aspecto y amplíen el campo de la reflexión científica y humanística.

\section{BILBIOGRAFÎA}

Bergesen, A. (2004). Chomsky versus Mead. Sociological Theory 22, 3, pp. 357-370.

Bernstein, R. (2010). The Pragmatic Turn. Malde, MA: Polity.

Blumer, H. (1969). Symbolic Interactionism. Perspective and Method. New Jersey: Prentice-Hall.

Booth, K. (2016). Imitation and Taking the Attitude of the Other. In H. Joas, \& D. Huebner, The Timeliness of George Herbert Mead (pp. 231-251). Chicago: University of Chicago Press.

Booth, K. (2016a). The Meaning of the Social Body: Bringing George Herbert Mead to Mark Johnson's Theory Of Embodied Mind. William James Studies 12, 1, pp. 1-18.

Brothers, L. (1997). Friday's Footprint. How Society Shapes the Human Mind. Oxford University Press: New York.

Clark, A. (1997). Being There: Putting Mind, Body, and World Together Again. Cambridge, MA: The MIT Press. 
Damasio, A. (1994). Descartes's Error. Emotion, Reason, and the Human Brain. New York: Avon Books.

Donald, M. (1991). Origins of the Modern Mind. Three Stages in the Evolution of Culture and Cognition. Cambridge, MA: Harvard University Press.

Engel, A., Friston, K., \& Kragic, D. (Eds.). (2015). The Pragmatic Turn. Toward ActionOriented Views in Cognitive Science. Cambridge, MA: The MIT Press.

Farnell, B. (1999). "Moving Bodies, Acting Selves". Annual Review of Anthropology, Vol. $28,341-373$.

Fodor, J. (1975). The Language of Thought. Cambridge, MA: Harvard University Press.

Fodor, J. (1980). "Methodological Solipsism Considered as a Research Strategy in Cognitive Science". Behavioral and Brain Sciences 3, 66-73.

Franks, D. (2010). Neurosociology. The Nexus between Neuroscience and Social Psychology. New York: Springer.

Gallagher, S. (2014). "Pragmatic Interventions into Enactive and Extended Conceptions of Cognition”. Philosophical Issues, 24, 1, pp. 110-126.

Gallagher, T. (2016). “G. H. Mead's understanding of the Nature of Speech in the Light of Contemporary Research". In H. Joas, \& D. Huebner, The Timeliness of George Herbert Mead (pp. 315-336). Chicago: The University of Chicago Press.

Gibson, J. (1979). The Ecological Approach to Visual Perception. Boston: Houghton Mifflin. Habermas, J. (1987). Teoría de la acción comunicativa, I. Madrid: Taurus.

Iacoboni, M., Molnar-Szakacs, I., Gallese, V., Buccino, G., Mazziotta, J., \& Rizzolatti, G. (2005). Grasping the Intentions of Others with One's Own Mirror Neuron System. PLoS Biol 3, 3, pp. 529-535.

Joas, H. (1985). G.H. Mead. A Contemporary Re-examination of his Thought. Massachussets: MIT Press.

Johnson, M. (2007). The Meaning of the Body. Chicago: University of Chicago Press.

Koczanowicz, L. (2011). "The Dialogical concept of consciousness in L.S. Vygotsky and G.H. Mead and its relevance for contemporary discussions on consciousness". Polish Psychological Bulletin, vol. 42, 2, pp. 65-70.

Lakoff, G., \& Johnson, M. (1980). Metaphors We Live By. Chicago: University of Chicago Press. Lakoff, G., \& Johnson, M. (1999). Philosophy in the Flesh, the embodied mind and its challenge to Western Thought. New York: Basic Books.

Lindblom, J. (2012). "Mead and socially embodied cognition: Reaping the Best of Both Worlds". In N. Payette, Connected Minds: Cognition and Interaction in the Social World (pp. 51-65). Cambridge: Cambridge Scholars Publishing.

Madzia, R. (2013). "Chicago Pragmatism and the Extended Mind Theory". European Journal of Pragmatism and American Philosophy 5, 1, pp. 279-297. 
Madzia, R. (2016). "Presentation and Re-Presentation: Language, Content, and the Reconstruction of Experience". In H. Joas, \& D. Huebner, The Timeliness of George Herbert Mead (pp. 296-314). Chicago: The University of Chicago.

Madzia, R., \& Jung, M. (Eds.). (2016). Pragmatism and Embodied Cognitive Science: From Bodily Intersubjectivity to Symbolic Articulation. Berlin: Walter de Gruyter.

McNeill, D. (2005). Gesture and Thought. Chicago: University of Chicago Press.

McVeigh, R. (2015). "Basic-Level Categories, Mirror Neurons, and Joint-Attention Schemes: Three Points of Intersection between G.H. Mead and Cognitive Science". Symbolic Interaction, 39, 1, pp. 45-65.

Mcveigh, R. (2016). "Mead, the Theory of Mind, and the Problem of Others". In H. Joas \& D. Huebner, The Timeliness of George Herbert Mead (pp. 209-230). Chicago: The University of Chicago Press.

Mead, G. H. (1894c). Review of "Die Moderne Energetik in ihrer Bedeutung für die Erkenntnisskritik" by Kurd Lasswitz. Psychological Review 1, pp. 210-213.

Mead, G. H. (1895). Review of "An Introduction to Comparative Psychology by C. Lloyd Morgan”. Psychological Review 2, pp. 399-402.

Mead, G. H. (1895b). "A Theory of Emotions from the Physiological Standpoint” (Abstract of a paper read to the third annual meeting of the American Psychological Association, 1894). Psychological Review, 2 (1895), pp. 162-164.

Mead, G. H. (1898). "The Child and His Environment". Transactions of the Illinois Society for Child-Study 3, pp. 1-11.

Mead, G. H. (1899a). “The Working Hypothesis in Social Reform”. American Journal of Sociology 5, pp. 367-371.

Mead, G. H. (1899b). Review of the Psychology of Socialism by Gustav Le Bon. American Journal of Sociology 19, pp. 404-412.

Mead, G. H. (1907). Concerning Animal Perception. Psychological Review 14, pp. 383-390.

Mead, G. H. (1932). The Philosophy of the Present. (A. Murphy, Ed.) Chicago: Open Court.

Mead, G. H. (1934). Mind, Self, and Society. From the Standpoint of a Social Behaviorist. (C. Morris, Ed.) Chicago: The University of Chicago Press.

Mead, G. H. (1936). Movements of Thought in the Nineteenth Century. (M. Moore, Ed.) Chicago: The University of Chicago Press.

Mead, G. H. (1938). The Philosophy of the Act. (C. Morris, Ed.) Chicago: The University of Chicago Press.

Mead, G. H. (1964). Selected Writings: George Herbert Mead. (A. Reck, Ed.) Chicago: University of Chicago Press.

Mead, G. H. (1982). The Individual and the Social Self. Unpublished Work of George Herbert Mead. (D. Miller, Ed.) Chicago: The University of Chicago Press.

Mead, G. H. (2001). Essays in Social Psychology. (M. J. Deegan, Ed.) New Brunswick: Transaction Publishers. 
Mead, G. H. (2001). “On Perception and Imitation”. In G. H. Mead, \& M. J. Deegan (Ed.), Essays in Social Psychology (pp. 67-72). New Brunswick: Transaction Publishers.

Mead, G. H. (2001). "Social Psychology as Counterpart to Physiological Psychology". In G. H. Mead, \& M. J. Deegan (Ed.), Essays on Social Psychology (pp. 9-17). New York: Routledge.

Mead, G. H. (2001). "The Relation of the Embryological Development to Education”. In G. H. Mead, \& M. J. Deegan (Ed.), Essays on Social Psychology (pp. 73-82). New York: Routledge.

Mead, G. H. (2001). “The Social Character of Instinct”. In G. H. Mead, \& M. J. Deegan (Ed.), Essays in Psychology (pp. 3-8). New York: Routledge.

Mead, G. H. (2011). G. H. Mead: A Reader. (F. C. da Silva, Ed.) New York: Routledge.

Mead, G. H. (2011). The Philosophy of Education. (G. Biesta, \& D. Troehler, Eds.) Boulder, CO: Paradigm Publishers.

Nungesser, F. (2016). "Mead Meets Tomasello: Pragmatism, the Cognitive Sciences, and the Origins of Human Communication and Socialit". In H. Joas, \& D. Huebner, The Timeliness of George Herbert Mead (pp. 252-275). Chicago: University of Chicago Press.

Piaget, J. (1980). The psychogenesis of knowledge and its epistemological significance. In M. Piatelli-Palmarini, Language and learning: The debate between Jean Piaget and (pp. 23-34). Cambridge, MA: Harvard University Press.

Puddephatt, A. (2011). "Language and Mind in The Thought of G. H. Mead: Challenges from Chomsky’s Linguistics”. Studies in Symbolic Interaction 36, pp. 75-106.

Rorty, R. (1979). Philosophy and the Mirror of Nature. Princeton: Princeton University Press.

Rosenthal, S., \& Bourgeois, P. (1991). Mead and Merleau-Ponty: Toward a Common Vision. New York: State University of New York Press.

Rowlands, M. (2010). The New Science of the Mind. From Extended Mind to Embodied Phenomenology. Cambridge, MA: The MIT Press.

Royce, J. (1895). "Self-consciousness, Social Consciousness and Nature (I)”. Philosophical Review 4, pp. 465-485.

Searle, J. (1969). Speech acts: An essay in the philosophy of language. Cambridge: Cambridge University Press.

Solymosi, T., \& Shook, J. (2013). "Neuropragmatism: A Neurophilosophical Manifesto". European Journal of Pragmatism and American Philosophy 5, 1, pp. 212-234.

Tomasello, M. (1999). The Cultural Origins of Human Cognition. Cambridge, MA: Harvard University Press.

Varela, F. J., Thompson, E. T., \& Rosch, E. (1991). The Embodied Mind: Cognitive Science and Human Experience. Massachusetts: MIT Press.

Weichold, M. (2017). "Enacting the Moral Self. Combining enactivist cognitive science with Mead's Pragmatism”. Pragmatism Today 8, 1, pp. 146-172. 